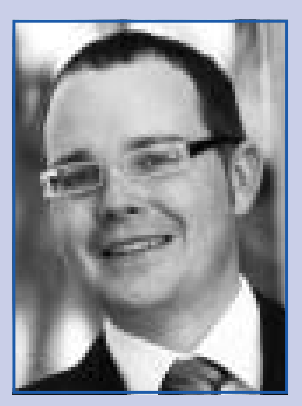

Dr. Christian Kluge ist Leiter Zentralbereich Controlling bei der Miele \& Cie. KG in Gütersloh.

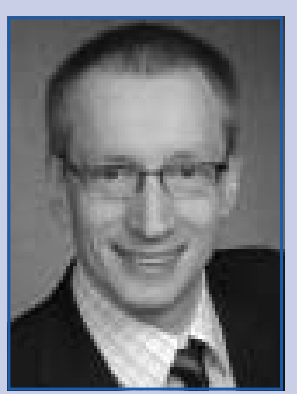

Prof. Dr. Andreas Hoffjan ist Inhaber des Lehrstuhls Unternehmensrechnung und Controlling an der Technischen Universität Dortmund sowie Mitherausgeber der Zeitschrift Controlling.

\title{
Controlling bei der Miele \& Cie. KG
}

\author{
Christian Kluge und Andreas Hoffjan
}

Miele ist der weltweit führende Anbieter von Premium-Hausgeräten für die Küche, Wäsche- und Bodenpflege. Hinzu kommen verschiedene Produkte für den gewerblichen Einsatz sowie für medizinische Einrichtungen und Laboratorien (Miele Professional). Das 1899 gegründete Unternehmen unterhält acht Produktionsstandorte in Deutschland sowie je ein Werk in Österreich, Tschechien, China und Rumänien. Der Umsatz betrug im Geschäftsjahr 2013/2014 rund 3,22 Mrd. Euro. Weltweit beschäftigt Miele rund 17.500 Menschen, zwei Drittel davon in Deutschland. Der Hauptsitz ist Gütersloh in Westfalen.

Andreas Hoffjan: Die Miele \& Cie. KG ist ein familiengeführtes Unternehmen in der vierten Generation. Familienunternehmen gelten allgemein als Rückgrat der Wirtschaft und weisen meist einen langfristigen strategischen Entscheidungshorizont auf. Wo bestehen aus Ihrer Sicht die zentralen Besonderheiten im Bereich Controlling?

Christian Kluge: Ein wesentlicher Punkt ist in der Tat, dass für ein Familienunternehmen wie Miele die nachhaltige Performance im Vordergrund steht, obwohl wir uns natürlich auch die Monatszahlen sehr genau ansehen. So akzeptieren wir auch längere Investitionsphasen in Produkte oder Märkte, wenn wir vom langfristigen Nutzen überzeugt sind. Das Controlling muss stimmig auf diese Unternehmensphilosophie ausgerichtet sein. Neben den Grundlagen - die richtigen Zahlen zur richtigen Zeit für jeden Bedarf zu erzeugen - geht es darum, zu erkennen, ob wir langfristig richtig unterwegs sind und bei Entscheidungen die richtige Balance zwischen monetären und nichtmonetären Argumenten zu finden. Darüber hinaus ist es unsere Aufgabe, die Unternehmenssteuerung so auszurichten, dass langfristige Performance gefördert wird und die Fraktionen in der Lösungssuche zusammengeführt werden.

Andreas Hoffjan: Diese Grundausrichtung hat vermutlich Auswirkungen auf die konkrete Ausgestaltung des Controllings. Welche Aufgaben werden durch das
Controlling bei Miele wahrgenommen und wie ist es dafür organisiert?

Christian Kluge: Die Basis bilden die Planung, die innerjährige Steuerung und das Reporting auf Konzernebene - mit Blick auf Organisationseinheiten, Produkte und große Projekte. Darüber hinaus organisiert das Controlling den Strategieprozess im Unternehmen und wirkt in der Strategiearbeit mit. Teil des Controllings ist auch das Rechnungswesen, das neben seinen klassischen Aufgaben in unserem Hause auch die internationale Standardisierung der Prozesse in unseren ERP-Systemen gestaltet. Außerdem kümmern wir uns um Risikomanagement und Versicherungen. Der Zentralbereich Controlling arbeitet eng mit über fünfzig dezentralen Controllingfunktionen in Vertriebsgesellschaften, Werken und Zentralfunktionen zusammen.

Andreas Hoffjan: Miele steht für hohe Qualität und langlebige Hausgeräte. Dies wird unter anderem dadurch deutlich, dass Sie als einziger Hersteller der Branche die Produkte auf 20 Jahre Lebensdauer testen. So hat sich das Unternehmen als Hersteller im Premiumpreissegment etabliert. Wie trägt das Controlling dazu bei, diese Positionierung langfristig beizubehalten?

Christian Kluge: Hier gibt es mehrere Ansatzpunkte. Einerseits geht es um alles, was uns erlaubt, die besten Produkte auf den Markt zu bringen. Das gesamte Instrumentarium der Entwicklungssteue- 
rung hilft dabei. Insbesondere ist Target Costing zu nennen, das es erlaubt, die Mittel so einzusetzen, dass der größte Kundennutzen entsteht. Andererseits geht es darum, ein gutes Produkt am Markt passend in Wert zu stellen. Hier sind wir beim Pricing. Miele Produkte sind z. T. deutlich teurer als der Wettbewerb, aber sie verkörpern auch einen höheren Wert. Gemeinsam mit dem Marketing sorgen wir dafür, dass die Preise zum Kundennutzen und zum Wert unserer Produkte passen. Bei Preisentscheidungen fließen nutzen- und kostenbasierte Indikationen sowie kurzund langfristige Erwägungen zusammen unter Berücksichtigung der Preisabstände zum Wettbewerb.

Andreas Hoffjan: Die hohe Qualität ist ein besonderes Merkmal der Miele Produkte. Wie ist die Zusammenarbeit zwischen dem Qualitätsmanagement und dem Controlling ausgestaltet? Welche sind aus Ihrer Sicht die entscheidenden Kennzahlen zur Qualitätsmessung?

Christian Kluge: Die Arbeit des Qualitätsmanagements beginnt präventiv bereits im Bereich der Produktentwicklung. Mit geeigneten Prüf- und Freigabeverfahren wird die Auslegung der Geräte auf ihre lange Lebensdauer abgesichert. Für die Beseitigung von Fehlern nach Auslieferung verwendet das Qualitätsmanagement Informationen, mit denen sich Qualitätsereignisse schnell auf ihre Auslöser zurückführen und abstellen lassen. Hierzu interessiert die Häufigkeit bestimmter Fehlerbilder im Feld, speziell auch in Bezug auf konkrete Produktionszeiträume. In aggregierter Form wird dies in Schichtliniendiagrammen abgebildet. Als übergreifende Kennzahlen betrachten wir im Controlling u. a. Garantie- und Kulanzkostenquoten und die Kundenausfallrate. Berührungspunkte zwischen beiden Funktionen liegen im inhaltlichen Austausch zum Qualitätsstatus je Produktbereich und in der Datenbeschaffung.

Andreas Hoffjan: In der Langzeitbetrachtung der Best Brands Awards, die im Rahmen einer jährlichen GfK-Studie verliehen werden, gilt Miele als die stärkste Produktmarke der letzten zehn Jahre. Somit erhielt das Unternehmen 2013 den Titel „Best Brand Ever“. Welchen Stellenwert hat in diesem Zusammenhang das Markencontrolling bei Miele?

Christian Kluge: Die Marke Miele verkörpert die Werte und das Leistungsver- sprechen des Unternehmens, aufgebaut über eine lange Historie von Kundenerfahrungen. Deshalb berücksichtigen wir bei Entscheidungen stets auch die Wirkung auf unsere Marke. Was der Marke schadet, kommt nicht in Betracht. Gleichzeitig müssen wir im Controlling natürlich darauf achten, dass dieses Argument nicht überstrapaziert wird. Davon abgesehen ist es wichtig, den Status unserer Marke zu beobachten. Dies ist eine Aufgabe unserer Marktforschung. Wichtige Beobachtungsgrößen sind dabei die Markenbekanntheit, die Markenbegehrlichkeit und die Kaufabsicht.

Andreas Hoffjan: Eine besondere Herausforderung großer Unternehmen ist die stark diversifizierte StakeholderStruktur. Dies wird bei Miele vor allem mit Blick auf die Kundensegmente deutlich. Diese beinhalten Privat- und Geschäftskunden, Händler sowie Architekten und Bauträger. Gibt es in Ihrem Unternehmen verschiedene Kundeninformationssysteme zur gezielten Steuerung der Kundenbearbeitung? Welche Aspekte sind in diesem Zusammenhang für den Bereich Controlling wichtig?

Christian Kluge: Wir müssen hier unterscheiden. Im operativen Geschäft sind Kundeninformationssysteme bei Miele ein Hilfsmittel für Marketing, Vertrieb und Kundendienst in ihrer Aufgabe, individuellen Kundennutzen zu generieren. Sie dienen z. B. dazu, bei einem Serviceanruf die Kommunikationshistorie sofort verfügbar zu machen. Aus Sicht des Controllings interessieren dagegen hierauf aufbauende aggregierte Erkenntnisse, z. B. zur Entwicklung einzelner Vertriebskanäle. Diese und andere Daten ermöglichen ein Verständnis über Veränderungen in der Vertriebslandschaft, was wichtig ist z. B. für die Lenkung von Marketing- und Vertriebsressourcen und für die Gestaltung eines leistungsgerechten Konditionensystems.

Andreas Hoffjan: Eine weitere Stakeholder-Gruppe bilden die Lieferanten, die vor allem aufgrund ihres Einflusses auf die Qualität eine entscheidende Bedeutung für das Unternehmen aufweisen. Welche Rolle spielt das Einkaufscontrolling bei Miele und welche Instrumente nutzen Sie zum Messen der Einkaufsaktivitäten?

Christian Kluge: Obwohl Miele eine vergleichsweise hohe Wertschöpfungstiefe hat, ist auch der Beitrag der Zulieferer entscheidend für die Qualität und für die Kosten unserer Produkte. Auch bei Miele stellen Materialkosten die größte einzelne Kostenposition in der GuV dar. Im Einkaufsbereich hat sich seit Längerem ein maßnahmenorientierter Controllingansatz bewährt. Hierbei werden einzelne konstruktive und kaufmännische Maßnahmen in einer Härtegradlogik verfolgt, und es werden aggregierte Gesamtaussagen je Materialgruppe und je Werk abgeleitet. Die Qualität wird in einem Instrumentarium zur Lieferantenbewertung überwacht, gemeinsam mit weiteren Leistungskriterien.

Andreas Hoffjan: Die Komplexität des Lieferantenmanagements und der Fertigungsprozesse wird durch die acht deutschen und vier internationalen Produktionsstandorte erhöht. Welche Instrumente zum Steuern Ihres Produktionsnetzwerkes ziehen Sie heran?

Christian Kluge: Bei Miele existiert eine ausgeprägte dezentrale Verantwortungskultur, sowohl in den Vertriebsgesellschaften als auch in den Werken. Steuerung besteht daher vor allem darin, den dezentralen Entscheidern die richtigen Informationen zur Verfügung zu stellen, damit im Sinne bestmöglicher Konzernperformance gehandelt werden kann. Hierfür blicken wir beispielsweise nicht auf isolierte Ergebniszahlen eines Werkes, sondern auf den übergreifenden Erfolg von Produktsparten. Durch dieses gemeinsame Verständnis lassen sich viele Fragen zwischen den Werken und zwischen Werk und Produktmanagement dezentral lösen. Im Controlling messen wir je Produktsparte sowohl den Geschäftserfolg als auch die einzelnen Erfolgsbeiträge anhand diverser Kennzahlen.

Andreas Hoffjan: Entsprechend einer Pressemitteilung aus Dezember 2013 wird Miele 23 Mio. Euro in den Logistikstandort Gütersloh investieren, um das Warenverteilzentrum zu erweitern. Die Kapazität soll um $75 \%$ erhöht werden. Welchen Stellenwert hat vor diesem Hintergrund das Investitionscontrolling bei Ihnen im Unternehmen?

Christian Kluge: Investitionscontrolling ist für uns ein Standardprozess mit dem Zweck, die Zielerreichung nachzuhalten und auf erkennbare Abweichungen richtig zu reagieren. An erster Stelle geht es 
um die Transparenz für den Projektverantwortlichen. Hier sind Mitarbeiter des zentralen oder dezentralen Controllings Dienstleister und auch Berater des Projektleiters. Der Projektleiter wiederum erstellt gesamthafte Statusberichte, die je nach Größe an verschiedene Adressaten gehen. Großprojekte werden vom zentralen Controlling begleitet. Hier blicken wir aus der Sicht der Geschäftsleitung auf das Projekt, sichern die Qualität der Statusinformationen und filtern die Themen heraus, die besondere Aufmerksamkeit verdienen.

Andreas Hoffjan: Mit der kürzlich erfolgten Umstellung der Modellgenerationen in den Bereichen Küchengeräte und Wäschepflege hat Miele erneut starke Produktinnovationen auf den Markt gebracht. Wie unterstützt das Controlling die Entwicklung von Innovationen bei Miele?

Christian Kluge: Das Feld der Innovationen erfordert im Controlling eine besondere Balance. Neben den angesprochenen Investitionen geht es dabei auch um Entwicklungskosten. Für Innovationsideen muss es kreative Freiräume geben, die wir auch gewährleisten. So können in einem definierten Rahmen Mittel reserviert werden, auch wenn noch kein Commitment zum Entwicklungserfolg abgegeben werden kann. Solche Aufgaben werden in Vorentwicklungsprojekten organisiert, bei denen auch größere Freiheitsgrade für Zielanpassungen bestehen. Auf der anderen Seite sind umfangreiche Schritte der Entwicklung relativ gut planbar. Für diese Teile, die auch für die Wirtschaftlichkeit des späteren Produkts ausschlaggebend sind, gelten strengere Maßstäbe für die Zielerreichung. Entscheidend für den Erfolg solcher Serienentwicklungsprojekte sind ein tragfähiges Zielgerüst und ein robuster Prozess der Zielführung. Aufgabe des Controllings ist es dabei nicht nur, die Zielerreichung nachzuhalten. Vielmehr geht es darum, beide Teilaufgaben durch Informationen und Methoden zu unterstützen, Zielkonflikte frühzeitig offenzulegen und für wirksame Regelkreise zu deren Lösung zu sorgen - im Schulterschluss mit allen beteiligten Funktionen.

Andreas Hoffjan: Miele ist Mitglied der UN-Nachhaltigkeitsorganisation Global Compact und veröffentlicht alle zwei Jahre einen Nachhaltigkeitsbericht, der auf Basis der Vorgaben der Global Reporting Initiative (GRI) erstellt wird. Im jüngsten Ranking des Instituts für ökologische Wirt- schaftsforschung (IÖW) wurde der Nachhaltigkeitsbericht aus dem Jahr 2011 auf Platz sieben geführt. Der aktuelle Bericht 2013 mit dem Titel „Denken in Generationen" ist noch ausführlicher und ambitionierter in den Zielen, und 2014 gewann Miele sogar den Deutschen Nachhaltigkeitspreis. Skizzieren Sie bitte kurz die Bedeutung des Themas Nachhaltigkeit für das Unternehmen Miele. Welche Berührungspunkte bestehen mit dem Bereich Controlling?

Christian Kluge: Aspekte der Nachhaltigkeit können unmittelbar erfolgsrelevant sein, etwa über die Marktattraktivität energieeffizienter Produkte. Sie sind es aber auch in mittelbarer Hinsicht, zum Beispiel im Sinne einer Rückkoppelung zwischen Unternehmensimage und Umsatz. Für Miele ist Nachhaltigkeit einer der zentralen Werte des Unternehmens. Der Erfolg des Nachhaltigkeitsberichts als bestes nicht im Dax notiertes Unternehmen - und erst recht die jüngst erhaltene hohe Anerkennung durch den Deutschen Nachhaltigkeitspreis wären nicht möglich, wenn diese Maxime nicht tatsächlich unser Denken und Handeln prägen würde. Aus Sicht des Controllings ist der Faktor Nachhaltigkeit vor allem bei der Bewertung von Entscheidungen relevant. Er ist aber nicht in vollem Umfang robust quantifizierbar, trotz diverser Ideen zu diesem Thema. Nachhaltigkeit ist daher für uns zumeist ein qualitatives Entscheidungskriterium, dass neben weiteren qualitativen Einschätzungen, z. B. über den Fit zur Marke, in die Bewertung einfließt und die quantitativen Aussagen zur Wirtschaftlichkeit ergänzt.

Andreas Hoffjan: Gibt es zwischen diesen Kriterien eine formale Zielhierarchie, nach der Sie sich richten?

Christian Kluge: Nein, die gibt es nicht. Nach unseren Erfahrungen sind auch die gängigen Methoden, z. B. die Nutzwertanalyse, nur dann zweckdienlich, wenn zwischen diversen Alternativen zu entscheiden oder eine komplexe Empfehlung zu vermitteln ist. In vielen Fällen liegt die Hauptaufgabe aber nicht im Vergleich, sondern in der Ausgestaltung guter Alternativen. Hierbei gilt es, Lösungen zu entwickeln, die in allen Feldern gute Ergebnisse liefern. Das Controlling versteht sich dabei als Business Partner aller Bereiche im Unternehmen - mit einer Fachaufgabe und einer unternehmerischen Position.

\section{Bezugspreise 2015}

\section{Controlling}

In Gemeinschaft mit dem Verlag Franz Vahlen.

Die Bezugspreise (einschl. MwSt.) betragen ab 1. Januar 2015:

Normalpreis jährlich € 209,-

Vorzugspreis für Bezieher der

Zeitschrift "BC« sowie Studenten fachbezogener Studiengänge (gegen Nachweis)

jährlich € 135,-

jeweils zuzüglich Vertriebs-।

Direktbeorderungsgebühren

jährlich (€ 10,80/€ 2,80)

$€ 13,60$.

Abbestellung bis 6 Wochen vor Jahresende.

Einzelheft $€ 22,-$

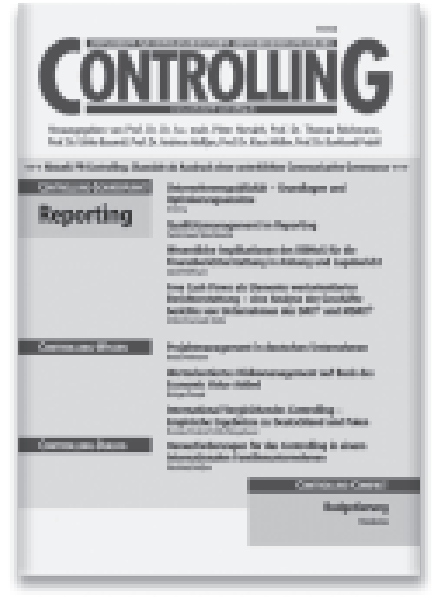

Bestellen Sie bei Ihrem Buchhändler oder bei: Bestellen Sie bei Ihrem Buchhändler oder bei:
beck-shop.de oder Verlag C.H.BECK · 80791 München Fax: 089/38189-358 - www.beck.de

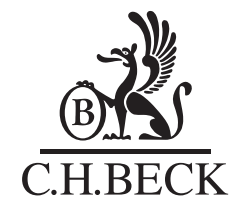

